\title{
A New Algorithm of SAR Image Target Recognition based on Improved Deep Convolutional Neural Network
}

\author{
Fei Gao ${ }^{1} \cdot$ Teng Huang ${ }^{1 *} \cdot$ Jinping Sun ${ }^{1} *$. \\ Jun Wang ${ }^{1}$. Amir Hussain ${ }^{2}$. Erfu Yang ${ }^{3}$
}

Received: date / Accepted: date

\begin{abstract}
Background: To effectively make use of deep learning technology automatic feature extraction ability, and enhance the ability of depth learning method to learn and recognize features, this paper proposed a deep learning algorithm combining Deep Convolutional Neural Network (DCNN) trained with an improved cost function and Support Vector Machine (SVM).

Methods: The class separation information, which explicitly facilitates intra-class compactness and interclass separability in the process of learning features, is added to an improved cost function as a regularization term to enhance the feature extraction ability of DCNN. Then the improved DCNN is applied to learn the features of SAR images. Finally, SVM is utilized to map the features into output labels.

Results: Experiments are performed on SAR image data in Moving and Stationary Target Acquisition and Recognition (MSTAR) database. The experiment results prove the effectiveness of our method, achieving an average accuracy of $99 \%$ on ten types of targets, some variants, and some articulated targets.

Conclusion: It proves that our method is effective and CNN enjoys a certain potential to be applied in SAR image target recognition.
\end{abstract}

Keywords synthetic aperture radar (SAR) images · automatic target recognition (ATR) - deep convolutional neural Network $(\mathrm{DCNN}) \cdot$ support vector machine $(\mathrm{SVM}) \cdot$ class separation information

\section{Introduction}

Synthetic Aperture Radar (SAR) possesses all-time and all-weather imaging capability even in harsh environments. As an important means of earth observation currently, it has been widely used in both military and civil fields. Automatic Target Recognition technology of SAR images (SAR-ATR), which can effectively obtain the target information and improve the ability of automatic information processing, has become one of the research hotspots.

SAR-ATR systems generally consist of three stages: detection [1, 2], recognition, and classification. At present, common methods of SAR-ATR include template matching [3], support vector machine (SVM)[4], Linear interpolation [5] Principal Component Analysis [6, 7], multi-modal dictionary learning and sparse representation combined $[8,9]$, etc. These methods have been successful in some way, but they rely heavily on experience of experts. The SAR image is vulnerable to various environmental factors, e.g., speckle noise and background clutter, leading to difficulties in the feature extraction of interested targets. In addition, shift sensitivity and pose sensitivity also cause instability in SAR targeting. Therefore, these methods have a certain blindness and unpredictability when applied to SAR images.

Recently, Deep Learning has been an increasingly hot topic in the field of pattern recognition, and experimental achievements have been emerging. In 2006, Hinton et al. [10] proposed an unsupervised greedily method based on Deep Belief Network (DBN), which trained deep networks layer by layer, solving the vanishing gradient problem. It also made the number of neural networks in a deeper direction with the help of GPU. After

\footnotetext{
* Corresponding Author, E-mail: huangteng1220@buaa.edu.cn, sunjinping@buaa.edu.cn, Tel.: 010-82317240,Fax: 010-8231724

${ }^{1}$ School of Electronic and Information Engineering, Beihang University, Beijing 100191, China

2 Cognitive Signal-Image and Control Processing Research Laboratory, School of Natural Sciences, University of Stirling, Stirling FK9 4LA, UK

${ }^{3}$ Space Mechatronic Systems Technology Laboratory, Department of Design, Manufacture and Engineering Management, University of Strathclyde, Glasgow G1 1XJ, UK
} 
that, many scholars proposed various deep learning models under different application backgrounds, such as the Deep Restricted Boltzmann (DRB)[11], Deep Belief Network (DBN) [12, 13], Stacked Auto Encoders (SAE) [14] and Deep Convolutional Neural Network (DCNN) [15]. Among them, some models based on DCNN have made breakthroughs endlessly in both theory and practice of image target recognition. For example, the AlexNet model, which won the championship in the contest of ImageNet ILSVRC in 2012, was recognized as the standard model of DCNN. The top-5 error rate of the AlexNet model was only 16\%, $10 \%$ lower than the last champion algorithm. The Residual Neural Network [16] won the championship in the same contest in 2015 and obtained only $3.75 \%$ error rate, which even outperformed human eyes. DCNN can not only automatically extract target features without too much experience of experts, but also deal with the two-dimensional image data directly. It means the features extracted by convolution kernel are irrelevant to the shift, scaling, and rotation of targets in images [17, 18]. This characteristic of DCNN provide a new idea to solve shift sensitivity, and pose sensitivity problems of SAR targets in automatic feature extraction.

Nowadays, the DCNN-based SAR-ATR system is being gradually proposed. To auto-learn features of SAR images rapidly, general methods start with the framework of AlexNet model. The promotion of training efficiency and recognition accuracy are achieved by optimizing a certain model. For instance, Chen et al. [19] initialize the hyper-parameters of DCNN with an unsupervised sparse auto encoder machine rather than the Back Propagation algorithm used in AlexNet model. The unsupervised sparse auto-encoder machine possesses the auto-learning capacity, which accelerates the feature learning of DCNN. The experiment of this method [19] on Moving and Stationary Target Acquisition and Recognition (MSTAR) database obtains a target recognition accuracy of $90.1 \%$ for 3 classes and $84.7 \%$ for 10 classes. Similarly, Li et al. [20] used the auto-encoder machine to initialize the DCNN. The difference is that fully connected layers work as a final classifier in [20], which greatly reduces training time of DCNN on the premise of ensuring accuracy. Although the accuracy of two methods is not very high, there is less dependence on experience of experts in the process of feature learning. Some scholars [21] apply DCNN to automatic feature learning as well, whereas the final output layer becomes a SVM classifier rather than a fully connected layer, constituting DCNN+SVM model. The model makes full use of the DCNNs superiorities in auto-learning of various features and the strong generalization ability of SVM, avoiding weak representation ability of SVM on high-dimensional samples [22] and poor stability of DCNN. With this framework, the classification accuracy of the method reaches $98.6 \%$ [21]. Because of these advantages of DCNN+SVM, such methods have been rapidly developed. On the basis of the work in [21], Wangner et al. [23] introduce Morphological Component Analysis to preprocess SAR image. It rejects some abnormal testing samples so that the accuracy reaches $99 \%$ and the recall comes up to $97.3 \%$.

Furthermore, shift sensitivity or pose sensitivity of targets can be solved by Data Augmentation technic, which adds speckle on training samples or extend training samples by shifting and rotating targets. For example, the SAR targets are rotated in [21] to acquire augmented training samples. Ding et al. [24] extend the MSTAR training samples by shifting, attitude synthesis, and adding speckles, achieving a higher accuracy on the standard DCNN methods. Chen et al. [25] augments training samples by shifting, and obtained a $99 \%$ classification accuracy using All-convolutional networks. A higher accuracy is acquired in [26] which extends classifier by displacement- and rotation-insensitive, and it is robust to shift and rotation. Whats more, Data Augmentation technic can suppress overfitting and advance recognition accuracy.

In summary, the SAR-ATR system based on DCNN has achieved varying degrees of success, but it is still in its in-fancy. The research is generally conducted from the perspectives of the exostructure architecture of DCNN or the Data Augmentation, yet little focus on the optimization of internal functions in DCNN. The CNN+SVM model proposed in [21], for instance, uses the quadratic function as error cost function. Although the classification accuracy is satisfactory, it would be time-consuming if the neurons make an obvious mistake during the training of DCNN [18]. Compared with error cost function, the Cross-Entropy is applied as the loss function in [24, 25, 27], while there is a lack of in-depth optimization. In order to improve the classification ability of CNN, we attempt to add class separability information to cross-entropy cost function as a regularization term in DCNN+SVM model.

The remainder of this paper is organized as follows. Section II gives an introduction of the basic principle of DCNN for a better understanding of our DCNN+SVM. Section III describes our DCNN+SVM in detail. Experimental results on the MSTAR database are presented in Section IV. Section V concludes our work.

\section{Deep Convolutional Neural Network}

DCNN is a kind of multilayer neural network, composed mainly of the input layer, convolutional layer, pooling layer and output layer. Among them, convolutional layer and pooling layer are hidden layers. In DCNN, the input layer firstly receives pixel values from the original input image. Then, the convolution layer extracts features from the image with convolution kernel. Afterward, the data needed to be processed is cut in the pooling layer according to local correlation principle. Finally, the output layer maps the feature to the labels. 
The mapping in DCNN is a forward propagation process which describes the "flow of information" through the whole neural network from its input layer to its output layer, so the output of the upper layer is actually the input of the current layer. To avoid the defects of linear model, neurons of each layer need to be added with a nonlinear activation function in the forward process. Since the first layer only receives pixel values from the image, there are no activation functions. The nonlinear activation functions are employed from the second layer and the output of the $l^{\text {th }}$ layer can be expressed as:

$$
\left.\begin{array}{l}
z^{l}=W^{l} * x^{l-1}+b^{l} \\
a^{l}=\sigma\left(z^{l}\right)
\end{array}\right\}
$$

where $l$ represents the $l^{\text {th }}$ layer, and $*$ means convolution operation. When $l=2, x^{2-1}=x^{1}$ is the image matrix whose elements are pixel values, and when $l>2, x^{l-1}$ is the feature map matrix extracted from the $(l-2)^{t h}$ layer i.e., $x^{l-1}=a^{l-1}=\sigma\left(z^{l-2}\right)$. $W^{l}, b^{l}$, and $z^{l}$ are weight matrix , bias matrix, and weighted input of the $l^{\text {th }}$ layer, respectively, and $\sigma$ is nonlinear activation function. Supposing $L$ is the output layer, $a^{L}$ will represent the final actual output vector.

The Back Propagation (BP) algorithm [28], which is a supervised learning method, is commonly used to iteratively update parameters of $W^{l}$ and $b^{l}$. It first applies the actual output and the targeted values to construct a cost function, and then applies the Gradient Descent (GD) along the negative gradient direction of cost function to adjust and parameters. The specific process is as follows:

Cost function selection. Normally the quadratic function is selected to be error cost function. However, it would be time-consuming if the neurons make an obvious mistake during the training of DCNN [18]. Thus we take Cross Entropy $\left(E_{0}{ }^{L}\right)$ rather than quadratic function as error cost function. According to the forward propagation algorithm (the equation (2)), the Cross Entropy can be inferred:

$$
E_{0}{ }^{L}=\frac{1}{n} \sum_{i=1}^{n} \sum_{k=1}^{N}\left[t_{k}^{L} \ln a_{k}^{L}+\left(1-t_{k}^{L}\right) \ln \left(1-a_{k}^{L}\right)\right]
$$

where $n$ is the total number of training set, and $N$ is the number of neurons in output layer, which means DCNN is finally divided into $N$ classes. $t_{k}^{L}$ is the targeted value corresponding to the $k^{\text {th }}$ neuron of output layer, and $a_{k}^{L}$ is the actual output value of the $k^{\text {th }}$ neuron of output layer.

Calculation of error vectors. The error vector of each layer is defined and the error vector corresponding to the $k^{\text {th }}$ neuron of output layer is expressed as:

$$
\delta^{L}=\frac{\partial E_{0}^{L}}{\partial z^{l}}
$$

In BP process, $\delta^{L}$ can be used to reversely deduce $\delta^{L}-1$. Similarly, suppose $\delta^{l}$ and $\left.\delta^{(} l+1\right)$ are the error vectors of the $l^{\text {th }}$ and $(l+1)^{\text {th }}$ layers respectively. Then according to (1), (3), and the Chain Rule, $\delta^{l}$ is written as:

$$
\delta^{l}=W^{l+1} \delta^{l+1} \odot \sigma^{\prime}\left(z^{l}\right)
$$

where the symbolic $\odot$ is Hadamard product (or Schur product) which denotes the elementwise product of the two vectors.

Updates of weights. The gradients of $W^{l}$ and $b^{l}$ are $\frac{\partial E_{0}^{L}}{\partial W^{l}}$ and $\frac{\partial E_{0}^{L}}{\partial b^{l}}$ respectively. The symbolic $\partial(\cdot)$ represents partial derivative operation. The partial derivative of $E_{0}^{L}$ to $W^{l}$ and $b^{l}$ can be calculated according to (1) and (3):

$$
\left.\begin{array}{l}
\frac{\partial E_{0}^{L}}{\partial W^{l}}=\frac{\partial E_{0}^{L}}{\partial a^{l}} \odot \frac{\partial a^{l}}{\partial W^{l}}=\delta^{l} \odot x^{l-1} \\
\frac{\partial E_{0}^{L}}{\partial b^{l}}=\frac{\partial E_{0}^{L}}{\partial a^{l}} \odot \frac{\partial a^{l}}{\partial b^{l}}=\delta^{l}
\end{array}\right\}
$$

The updated values of $W^{l}$ and $b^{l}$ are represented with $\Delta W^{l}$ and $\Delta b^{l}$, and they are calculated by minimizing the cost function respectively:

$$
\left.\begin{array}{l}
\Delta W^{l}=-\eta \frac{\partial E_{0}^{l}}{\partial W^{l}} \\
\Delta b^{l}=-\eta \frac{\partial E_{0}^{l}}{\partial b^{l}}
\end{array}\right\}
$$

where $\eta$ represents learning rate. 


\section{Improvement of Deep Convolutional Neural Network}

3.1 The introduction of class separability information

To enhance the class separability of the features extracted by DCNN model, the class separability information is added to cross-entropy cost function as a regularization term to train DCNN. The class separability information consists of intra-class compactness and inter-class separability, which are expressed as $E_{1}$ and $E_{2}$ respectively:

$$
\begin{gathered}
E_{1}=\frac{1}{2} \sum\left\|y_{c}^{n}-M_{c}\right\|_{2}^{2} \\
E_{2}=\frac{1}{2} \sum_{c^{\prime}}^{B}\left\|M_{c}-M_{c^{\prime}}\right\|_{2}^{2}
\end{gathered}
$$

where $y_{c}^{n}$ denotes the actual output value of the $n^{\text {th }}$ training sample which belongs to the $n^{\text {th }}$ class. $M_{c}$ and $M_{c}$ are the output average values of the training samples in $c^{\text {th }}$ and $\left(c^{\prime}\right)^{\text {th }}$ classes. $B$ is the number of training samples in $\left(c^{\prime}\right)^{\text {th }}$ classes. $E_{1}$ and $E_{2}$ respectively denote the intra-class distance and the inter-class distance of the output features. Shortening the intra-class distance and increasing the inter-class distance in every iteration are necessary to enhance the separability of the output features. It is added in the cost function as a regularization term and the modified cost function is as follows:

$$
E=E_{0}{ }^{L}+\alpha E_{1}-\beta E_{2}
$$

where $\alpha$ and $\beta$ are both weight parameters.

The purpose of modifying the cost function is to adjust $W^{l}$ and $b^{l}$ to make the network develop in favor of classification, and the error vector of modified cost function is essential.

The error vector of $E_{1}$ in the output layer is:

$$
\begin{aligned}
\delta_{1}{ }^{L} & =\frac{\partial E_{1}}{\partial z^{L}} \\
& =\frac{\partial}{\partial z^{L}} \frac{1}{2}\left\|y_{c}^{n}-M_{c}\right\|_{2}^{2} \\
& =\left(1-\frac{1}{n^{c}}\right) \sigma^{\prime}\left(z^{L}\right) \odot\left(y_{c}^{n}-M_{c}\right)
\end{aligned}
$$

where $n^{c}$ is the number of samples that belong to the class $c$.

The error vector of $E_{2}$ in the output layer $L$ is:

$$
\begin{aligned}
\delta_{2}{ }^{L} & =\frac{\partial E_{2}}{\partial z^{L}} \\
& =\frac{\partial}{\partial z^{L}} \frac{1}{2}\left\|M_{c}-M_{c^{\prime}}\right\|_{2}^{2} \\
& =\frac{1}{n^{c}} \sigma^{\prime}\left(z^{L}\right) \odot \sum_{c^{\prime}}^{B}\left(M_{c}-M_{c^{\prime}}\right)
\end{aligned}
$$

According to (3), (9), (10), and (11), the new error vector of $E$ in the output layer $L$ is:

$$
\begin{aligned}
\delta^{\prime}= & \delta^{L}+\alpha \delta_{1}{ }^{L}-\beta \delta_{2}{ }^{L} \\
= & \sigma^{\prime}\left(z^{L}\right) \odot\left(y^{n}-t^{n}\right)+\alpha\left(1-\frac{1}{n^{c}}\right) \sigma^{\prime}\left(z^{L}\right) \odot\left(y_{c}{ }^{n}-M_{c}\right) \\
& -\beta \frac{1}{n^{c}} \sigma^{\prime}\left(z^{L}\right) \odot \sum_{c^{\prime}}^{B}\left(M_{c}-M_{c^{\prime}}\right)
\end{aligned}
$$

After getting the error vector in the output layer, we can calculate the error vector in each layer iteratively by using (4). The updated parameters of $W^{l}$ and $b^{l}$ for each layer can be derived by (5) and (6) afterwards.

\subsection{Application of Support Vector Machines (SVM)}

In standard DCNN model, Softmax Regression model is usually applied as the classifier. It maps the extracted features into the output classes by the fully connected mode. Such methods can be effectively combined with the BP process, which facilitates the update of the parameters. However, the problem of nonlinear classification is still left to be solved. SVM implicitly maps the nonlinear classification interface of the original feature space to the higher dimensional feature space by kernel function to generate a linear classification interface. It works well in solving nonlinear classification problems. 


\subsection{Modified Model}

A novel DCNN+SVM model of SAR image target recognition is proposed based on above analysis. Firstly, DCNN is trained combining with the Softmax classifier, where the Cross-Entropy added with the class separability information is used as the cost function. After training the DCNN, Softmax is removed and the top features of DCNN is utilized to train SVM. Finally, the proposed framework DCNN+SVM is constructed, where DCNN is used to extract sample features and SVM is used as the classifier, as is shown in Figure 1.

\section{Experiment}

\subsection{Experiment data}

In order to verify the validity of the proposed method, this paper uses the data from the MSTAR database, cofunded by National Defense Research Planning Bureau (DARPA) and the U.S. Air Force Research Laboratory (AFRL). As a benchmark data set, MSTAR is widely applied for development, test, and evaluation of advanced ATR systems [29]. Therefore, adopting the MSTAR data is convenient for comparison with peer algorithms.

The MSTAR database includes different types of targets and their various serial numbers variant (vehicles that are of the same class and version, but are different serial numbers), version variant (differences from the manufacturer, target of same class but were built to different blueprint), articulation, aspect angles and depression angles. Figure 2 shows detailed parameters of MSTAR database. The size of each target chip is $128 * 128$, and the resolution is $0.3 \mathrm{~m} * 0.3 \mathrm{~m}$. The aspect angle covers from $0^{\circ}$ to $360^{\circ}$, and the interval is $1^{\circ}$ to $5^{\circ}$, which means there is one SAR image for every $1^{\circ}$ to $5^{\circ}$. This paper applies ten types of targets: 2S1, ZSU234, BMP2, BRDM2, BTR60, BTR70, D7, ZIL131, T62, and T72. As for their high-level classifications, 2 S1 and ZSU234 are artillery; BMP2, BRDM2, BTR60, BTR70, D7, and ZIL131 are assigned to truck; T62 and T72 belong to tank. Their SAR image samples and corresponding optical images are shown in Figure 3. The experiment is performed on the above ten types of targets, some other variants, and some articulated targets.

In order to explain our method more clearly and evaluate the performance of our method in a comprehensive way, the experiment is carried out under standard operating conditions (SOC) and extended operating conditions (EOC). The SOC means that the testing conditions and the training conditions are very similar [29], for example, 10 types of targets under $17^{\circ}$ depression are taken as the training samples while the ones under $15^{\circ}$ depression are used as testing samples. However, the EOC means that there are huge dissimilarities between the training set and the testing set [29], such as the significantly different depression angles, different variants of the same target, etc. The Correct Class Probability $\left(P_{c c}\right)$ is applied as the evaluation index of these experiments, and its expression is as follows [30]:

$$
P_{c c}=\frac{n_{c c}}{n_{t t}}
$$

Where $n_{c c}$ is the number of targets classified correctly, $n_{t t}$ is the total number of tested targets. In general, $P_{c c}$ is often presented combining with the confusion matrix.

\subsection{Experiments under SOC}

A This experiment aims to classify ten types of targets under SOC. The ten types of vehicle target chips at $17^{\circ}$ and $15^{\circ}$ depression angles mentioned in Section 4.1 are used as a training set and testing set respectively. The detailed information of type, size, and amount is shown in Table 1. In order to reduce the input dimension, $64 * 64$ pixels in the central part of the chip is intercepted as the input sample under the premise that the complete target still locates at the central position. With above data, this experiment is divided into two groups. One only uses the original data as train dataset, while another extends train dataset by Data Augmentation. The DCNN+SVM model in [19] and the DCNN+Softmax model in [27] are used as the contrast method.

The parameters of our model in this paper are set as follows. This model includes the input layer, the output layer, 4 convolutional layers and 2 pooling layers. Generally, a convolutional layer and a pooling layer are together regarded as a convolutional layer, so there are 6 layers in total. The first convolutional layer Conv (other layers follow the labeling rule) contains 18 neurons, and the size of convolution kernel and pool are set to $9 * 9$ and $7 *$ 7 respectively. After processing by convolutional kernel and pool, the $8 * 8$ feature map is output. For 2 nd Conv, the size of its convolution kernel is $5 * 5$ and the number of neurons is 120 , and a $2 * 2$ feature map is outputted after processing by the $5 * 5$ convolutional layer. Therefore, the size of the final feature map is $1 * 1$. Besides, 
ReLu is used as the activation function, and the initial bias and the learning rate are set to 0 and 1 respectively. The initial weight value of each layer is generated randomly in following interval:

$$
\left[-4 \sqrt{\frac{6}{{f a n_{\text {in }}}+\text { fan }_{\text {out }}}}, 4 \sqrt{\frac{6}{f a n_{\text {in }}+\text { fan }_{\text {out }}}}\right]
$$

where $f_{a n}$ in is the number of input feature map of each layer. $f_{a n}$ is the number of output feature map of each layer. Other parameters, $\alpha$ and $\beta$ in (9), are set to $\alpha=0.03$ and $\beta=2$.

\subsubsection{SOC_1:The data in Table 1 is used, where there is no extended data.}

The experimental results of the proposed method are shown in Table 2. Table 3 gives the results of contrast method, which applies the standardized quadratic cost function in the training process combining with Softmax and uses Softmax as a final classifier [19] (here only recapitulating its sequence). The average accuracy of our method is $97.84 \%, 13.14 \%$ higher than that of DCNN+Softamx. As for inter-class error, we find from Table 2 that only one truck is wrongly classified into tanks. The intra-class confusion in Table 2 is overall satisfactory, with no confusions in tanks, only one in artilleries, and slightly more in trucks. The confusions of BTR70 and BTR60 are relatively more, which may be caused because BTR70 is an upgraded version of BTR60. For DCNN+Softmax model, however, there are serious confusions in both inter and intra classifications. It proves that adding the class separability information to Cross Entropy error cost function is conducive for distinguishing the high-level classes.

\subsubsection{SOC_2:The data in this experiment is augmented by rotating and randomly shifting.}

Shifting:The number of extended samples of each chip can be increased up to $(128-64+1) *(128-64+1)=$ 4225 by shifting in case that every sample contains a complete target. An operation sample is shown in Figure 4.

Rotating:We apply the method in [26] to extend training samples, which rotates each chip every 24 degrees. Thus 15 extended chips are generated for each chip.

Combining the rotation and the random shift operation, each chip can be expanded to $4225 * 15$ chips at most. We select 3000 chips for each type of vehicle targets from the extended dataset. Then the same operation as experiment SOC_1 is carried out on the extended data. Table 4 shows the experimental results of the proposed method. The average accuracy in Table 4 reaches $99.15 \%$, better than that in Table 2 . There is no error in highlevel classification, and confusion only appears in trucks for intra-class classification. Compared with the results of experiment $\mathrm{SOC}_{-} 1$, the intra-class confusion in trucks reduces a lot in this experiment, and the accuracy is further enhanced as well.

As is introduced in the introduction section, the classification model in [21] is very similar with ours. The distinction lies in that we adopt the Cross-Entropy added with class separability information as the cost function rather than quadratic function. Since the experimental results in [21] do not include detailed information of ten types of targets, we only care about the average accuracy. Their average accuracy is $98.6 \%, 0.55 \%$ lower than ours. The DCNN+Softmax model in [27] employs Cross Entropy as cost function, and its experimental results are given in Table 5. Compared with Table 4, we find that there are more errors between high-level classes and more confusions between intra classes in Table 5. It proves that our cost function increases the class separability to some extent, especially for high-level classes.

\subsection{Experiments under EOC}

In this section, the experiment is performed under Extended Operating Condition (EOC), which is crucial to determine whether the ATR algorithm can be applied on the battlefield. To verify the reliability, stability, and the generalization ability of our method, we divide the experiment into 4 groups according to various experimental data in Figure 2. It is noted that each training samples of EOC series experiment is expanded to 3000 by the method described in section 4.2.2.

\subsubsection{EOC_1: The depression angles of the training set and the testing set are significantly different.}

We select 2S1 (B_01), BRDM2 (E_71), T72 (\#A64), and ZSU234 (D_08) four types of vehicle targets in this experiment, where the depression angles for the training set and testing set are $17^{\circ}$ and $30^{\circ}$ respectively. Table 6 shows the detailed data and Table 7 gives the experimental results of our method. It can be found from Table 7 that 
an accuracy of $99.57 \%$ is achieved, whats more, no error appears in high-level classes and only 5 confusions exist in the intra-class of artilleries. Thus we can conclude that our method works stably though the gaps of depression angles between the training set and testing set are large.

\subsubsection{EOC_2: Some articulated targets are contained in the testing set.}

Articulation means some parts of a vehicle change from one state to another, such as a newly added fuel tank or a barrel in different direction [31]. An articulated sample of ZSU234 is shown in Figure 5. It is essential to perform this experiment for articulated targets commonly appear in battlefield. To better simulate the practical conditions, we add some articulated targets into the training set and testing set, meanwhile, we keep $17^{\circ}$ and $30^{\circ}$ for the training set and testing set. For each high-level class, we select only one type of target in this experiment, and the detailed experimental data is given in Table 8. Table 9 shows the experiment results, and we find the accuracy of each class reaches $100 \%$. Therefore, our method behaves well when articulated targets are included in testing samples.

\subsubsection{EOC_3: The testing set contains one type of vehicle and its version variants, and the depression angle is not single.}

BMP2, BRDM2, one type of version variants of T72 (\#64), and ZSU234 consist of a training set, while the testing set only contains T72 and its version variants ( \#A04, \#A05, \#A07, \#A10, \#A32). The version variants belong to the same class, but the manufacturers are different, and the blue prints of manufacturing are different as well [30]. The variants mentioned above are shown in Figure 6. The depression angle of the training set is $17^{\circ}$, while the testing set has a $15^{\circ}$ depression angle apart from $17^{\circ}$. Table 10 shows the detailed data information. From Table 11, we find that testing accuracy is up to $100 \%$, so the version variants of T72 cannot impact high-level classification.

4.3.4 EOC_4: The testing set contains multi types of vehicles and their variants, and the depression angle is not single.

The training set is the same as (4.3.3) under EOC_3, while the testing set contains two types of version variants of T72 (\#A62, \#A63) and two types of serial number variants of BMP2 (SN_9566, C_21). The detailed data is shown in Table 12, and the experimental results are given in Table 12. The accuracy reaches $99.82 \%$, and there is a small number of confusions only in the trucks. Hence the version variants of T72 will not impact high-level classification, and the impact of serial number variants is also very little.

\subsection{Comparison with other algorithms}

In order to compare with the performance of other algorithms, this paper conducts the comparison from two aspects. One is the comparison with the algorithms in recent published papers, the other is the comparison with some "CNN+ Classifier" models.

Table 14 shows the comparison sheet of classification accuracy of recent published papers. The involved algorithms include Extend Maximum Average Correlation Height (EMACH) [32], Iterative Graph Thickening (IGT) [33], Sparse Representation of Monogenic Signal(MSRC) [34], Monogenic, Scale Space (MSS) [35] and Modified Polar Mapping Classifier (M-PMC)[36]. From Table 14, we find the accuracy of the proposed method in this paper is higher than other algorithms.

To constitute "CNN+Classifier" model, we select Softmax, RandomForest, AdaBoost and Bagging as classifiers, which are popular and superior classifiers among current machine learning models. The configuration of these models is shown in Table 15. The experiment is performed under both SOC and EOC. As for SOC, the experiment data and condition are the same as SOC_2. For EOC, the experiment is divided into 4 parts, whose training set and testing set follow the data in Table 6, Table 8, Table 10, and Table 12, respectively. These four kinds of data conditions are represented in order with EOC_1, EOC_2, EOC_3, and EOC_4. The recognition accuracy comparison sheet of these four structures is shown in Table 16. From Table 16, it can be found that the classification results of these five models are marvelous, all above 94\%, but CNN+SVM is still better than the other four models. Therefore, we can draw the conclusion that the modified cost function and Data Augmentation technic can significantly inhibit the overfitting phenomenon and solve the problem of the scarcity of training data. 


\section{Conclusion}

Aiming at the issue of SAR image target recognition, this paper presents a novel method of SAR image target recognition based on CNN. The CNN is improved by adding the class separability information into Cross Entropy cost function and applying support vector machine (SVM) instead of Softmax classifier. The experimental results show that the proposed method achieves a recognition accuracy of $99.15 \%$ for ten types of targets with extended training data. In other experiments under EOC, the average recognition accuracy can reach more than $99 \%$. It, therefore, proves that our method is effective and CNN enjoys a certain potential to be applied in SAR image target recognition.

\section{Acknowledgements}

This work was supported by the National Natural Science Foundation of China $(61771027 ; 61071139 ; 61471019$; 61671035). Dr E. Yang is supported in part under the RSE-NNSFC Joint Project (2017-2019) (6161101383) with China University of Petroleum (Huadong). Professor A. Hussain is supported by the UK Engineering and Physical Sciences Research Council (EPSRC) grant no. EP/M026981/1.

\section{Compliance with Ethical Standards}

Conflict of Interest: Author Fei Gao et al. declare that they have no conflict of interest. Ethical approval: This article does not contain any studies with human participants performed by any of the authors.

\section{References}

1. Gao, F., Ma, F., Zhang, Y., Wang, J., Sun, J., Yang, E., Hussain, A.: Biologically inspired progressive enhancement target detection from heavy cluttered sar images. Cognitive Computation 8(5) (2016) 1-12

2. Gao, F., Zhang, Y., Wang, J., Sun, J., Yang, E., Hussain, A.: Visual attention modelbased vehicle target detection in synthetic aperture radar images: A novel approach. Cognitive Computation 7(4) (2015) 434444

3. Owirka, G.J., Verbout, S.M., Novak, L.M.: Template-based sar atr performance using different image enhancement techniques. Proc Spie 3721 (1999) 302-319

4. Zhao, Q., Principe, J.C.: Support vector machines for sar automatic target recognition. IEEE Transactions on Aerospace \& Electronic Systems 37(2) (2001) 643-654

5. Ren, J., Jiang, J., Vlachos, T.: High-accuracy sub-pixel motion estimation from noisy images in fourier domain. IEEE Trans Image Process 19(5) (2010) 1379-1384

6. Zabalza, J., Ren, J., Yang, M., Zhang, Y., Wang, J., Marshall, S., Han, J.: Novel folded-pca for improved feature extraction and data reduction with hyperspectral imaging and sar in remote sensing. Isprs Journal of Photogrammetry \& Remote Sensing 93(7) (2014) 112-122

7. Zabalza, J., Ren, J., Ren, J., Liu, Z., Marshall, S.: Structured covariance principal component analysis for real-time onsite feature extraction and dimensionality reduction in hyperspectral imaging. Applied Optics 53(20) (2014) 4440

8. Lin, C., Wang, B., Zhao, X., Pang, M.: Optimizing kernel pca using sparse representation-based classifier for mstar sar image target recognition. Mathematical Problems in Engineering,2013,(2013-5-2) 2013(6) (2013) 707-724

9. Liu, H., Li, S.: Decision fusion of sparse representation and support vector machine for sar image target recognition. Neurocomputing 113(7) (2013) 97-104

10. Hinton, G.E., Salakhutdinov, R.R.: Reducing the dimensionality of data with neural networks. Science 313(5786) (July 2006) 504-507

11. Han, J., Zhang, D., Cheng, G., Guo, L., Ren, J.: Object detection in optical remote sensing images based on weakly supervised learning and high-level feature learning. IEEE Transactions on Geoscience \& Remote Sensing 53(6) (2015) 3325-3337

12. Montufar, G., Ay, N.: Refinements of universal approximation results for deep belief networks and restricted boltzmann machines. Neural Computation 23(5) (2011) 1306

13. Won, S., Young, S.S.: Assessing the accuracy of image tracking algorithms on visible and thermal imagery using a deep restricted boltzmann machine. Proc Spie 8401(7) (2012) 6 
14. Zabalza, J., Ren, J., Zheng, J., Zhao, H., Qing, C., Yang, Z., Du, P., Marshall, S.: Novel segmented stacked autoencoder for effective dimensionality reduction and feature extraction in hyperspectral imaging. Neurocomputing 214(C) (2016) 1062

15. Sun, M., Zhang, D., Ren, J., Wang, Z., Jin, J.S.: Brushstroke based sparse hybrid convolutional neural networks for author classification of chinese ink-wash paintings. In: IEEE International Conference on Image Processing. (2015) 626-630

16. He, K., Zhang, X., Ren, S., Sun, J.: Deep residual learning for image recognition. (2015) 770-778

17. Lecun, Y., Bengio, Y., Hinton, G.: Deep learning. Nature 521(7553) (2015) 436

18. Theodoridis, S.: Neural Networks and Deep Learning. (2015)

19. Chen, S., Wang, H.: Sar target recognition based on deep learning. In: International Conference on Data Science and Advanced Analytics. (2015) 541-547

20. Li, X., Li, C., Wang, P., Men, Z., Xu, H.: Sar atr based on dividing cnn into cae and snn. In: Synthetic Aperture Radar. (2015) 676-679

21. Wagner, S.: Combination of convolutional feature extraction and support vector machines for radar atr. In: International Conference on Information Fusion. (2014) 1-6

22. Huang, F.J., Lecun, Y.: Large-scale learning with svm and convolutional for generic object categorization. In: IEEE Computer Society Conference on Computer Vision and Pattern Recognition. (2006) 284-291

23. Wagner, S.: Morphological component analysis in sar images to improve the generalization of atr systems. In: International Workshop on Compressed Sensing Theory and ITS Applications To Radar, Sonar and Remote Sensing. (2015) 46-50

24. Ding, J., Chen, B., Liu, H., Huang, M.: Convolutional neural network with data augmentation for sar target recognition. IEEE Geoscience \& Remote Sensing Letters 13(3) (2016) 364-368

25. Chen, S., Wang, H., Xu, F., Jin, Y.Q.: Target classification using the deep convolutional networks for sar images. IEEE Transactions on Geoscience \& Remote Sensing 54(8) (2016) 4806-4817

26. Du, K., Deng, Y., Wang, R., Zhao, T., Li, N.: Sar atr based on displacement- and rotation-insensitive cnn. Remote Sensing Letters 7(9) (2016) 895-904

27. Kreucher, C.: Modern approaches in deep learning for sar atr. In: Algorithms for Synthetic Aperture Radar Imagery XXIII. (2016) 98430N

28. Pathak, G., Singh, B., Panigrahi, B.K.: Back propagation algorithm based controller for autonomous wind-dg microgrid. IEEE Transactions on Industry Applications 52(5) (2016) 4408-4415

29. Mossing, J.C., Ross, T.D.: Evaluation of sar atr algorithm performance sensitivity to mstar extended operating conditions. Proceedings of SPIE - The International Society for Optical Engineering 3370 (1998) 13

30. Ross, T.D., Velten, V.J., Mossing, J.C.: Standard sar atr evaluation experiments using the mstar public release data set. In: Algorithms for Synthetic Aperture Radar Imagery V. (1998) 566-573

31. Iii, G.J., Bhanu, B.: Recognizing articulated objects in sar images. Pattern Recognition 34(2) (2001) 469-485

32. Singh, R., Kumar, B.V.: Performance of the extended maximum average correlation height (emach) filter and the polynomial distance classifier correlation filter (pdccf) for multiclass sar detection and classification. Proceedings of SPIE - The International Society for Optical Engineering 4727 (2002) 265-276

33. Srinivas, U.: Sar automatic target recognition using discriminative graphical models. In: IEEE International Conference on Image Processing, ICIP 2011, Brussels, Belgium, September. (2014) 33-36

34. Dong, G., Wang, N., Kuang, G.: Sparse representation of monogenic signal: With application to target recognition in sar images. IEEE Signal Processing Letters 21(8) (2014) 952-956

35. Dong, G., Kuang, G.: Classification on the monogenic scale space: Application to target recognition in sar image. IEEE Transactions on Image Processing 24(8) (2015) 2527-2539

36. Park, J.I., Kim, K.T.: Modified polar mapping classifier for sar automatic target recognition. IEEE Transactions on Aerospace \& Electronic Systems Aes 50(2) (2014) 1092-1107 


\section{Tables}

\section{List of Tables}

1 The training and testing set of $\mathrm{SOC}_{-} 1 \ldots \ldots \ldots \ldots \ldots \ldots$

2 The experimental results of our method under SOC_1 f . . . . . . . . . . . . . . . . 10

3 The experimental results with the method in Literature $[19]$. . . . . . . . . . . . . . . . . 11

4 The experimental results of our method under SOC_2 . . . . . . . . . . . . . . . 11

5 The experimental results with the method in Literature $[27]$. . . . . . . . . . . . . . . . . 11

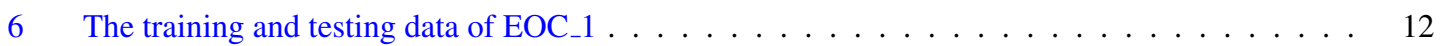

7 The experimental results of our method under EOC_1 . . . . . . . . . . . . . . . . 12

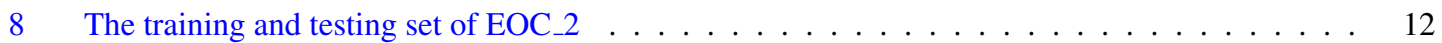

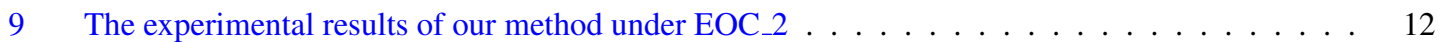

10 The training and testing set data of EOC_3 . . . . . . . . . . . . . . . . . . . 12

11 The experimental results of our method under EOC_3 . . . . . . . . . . . . . . . . 13

12 Training set and test set parameter configuration (EOC_4) for the experiment on the classification of mixed depression angle of variant of multi models and multi targets . . . . . . . . . . . 13

13 The experimental results of our method under EOC_4 . . . . . . . . . . . . . . . . . 13

14 Comparison sheet of classification accuracy between Our method and other algorithms . . . . 13

15 "CNN+Classifier" model parameter configuration . . . . . . . . . . . . . . . . . . . . . 14

16 Comparison of experimental results of "CNN+Classifier" models in Table 15 under SOC and

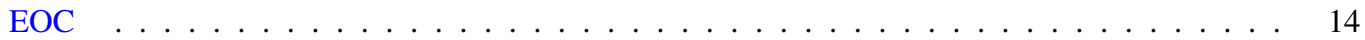

Table 1: The training and testing set of SOC_1

\begin{tabular}{|c|c|c|c|c|c|c|c|}
\hline \multirow{2}{*}{ Types } & \multirow{2}{*}{ Tops } & \multirow{2}{*}{ Variant } & \multicolumn{2}{|c|}{ Training set } & \multicolumn{2}{|c|}{ Testing set } & \multirow{2}{*}{ Image size } \\
\hline & & & depression angle & number & depression angle & number & \\
\hline 2S1 & \multirow{2}{*}{ artillery } & B_01 & $17^{\circ}$ & 299 & $15^{\circ}$ & 274 & $64 * 64$ \\
\hline ZSU234 & & D_08 & $17^{\circ}$ & 299 & $15^{\circ}$ & 274 & $64 * 64$ \\
\hline BRDM2 & \multirow{6}{*}{ truck } & E_71 & $17^{\circ}$ & 298 & $15^{\circ}$ & 274 & $64 * 64$ \\
\hline BTR60 & & K10YT & $17^{\circ}$ & 256 & $15^{\circ}$ & 195 & $64 * 64$ \\
\hline BMP2 & & SN_9563 & $17^{\circ}$ & 233 & $15^{\circ}$ & 195 & $64 * 64$ \\
\hline BTR70 & & C_71 & $17^{\circ}$ & 233 & $15^{\circ}$ & 196 & $64 * 64$ \\
\hline D7 & & $92 \mathrm{~V}$ & $17^{\circ}$ & 299 & $15^{\circ}$ & 274 & $64 * 64$ \\
\hline ZIL131 & & E_12 & $17^{\circ}$ & 299 & $15^{\circ}$ & 274 & $64 * 64$ \\
\hline T62 & \multirow{3}{*}{$\operatorname{tank}$} & A_51 & $17^{\circ}$ & 299 & $15^{\circ}$ & 273 & $64 * 64$ \\
\hline \multirow[t]{2}{*}{$\mathrm{T} 72$} & & \#A64 & $17^{\circ}$ & 299 & $15^{\circ}$ & 274 & $64 * 64$ \\
\hline & & & \multicolumn{2}{|c|}{ sum: 2814} & \multicolumn{2}{|c|}{ sum: 2503} & \\
\hline
\end{tabular}

Table 2: The experimental results of our method under SOC_1

\begin{tabular}{|c|c|c|c|c|c|c|c|c|c|c|c|}
\hline \multirow{2}{*}{ Types } & \multicolumn{2}{|c|}{ artillery } & \multicolumn{6}{|c|}{ truck } & \multicolumn{2}{|c|}{$\operatorname{tank}$} & \multirow{2}{*}{$P_{c c}(\%)$} \\
\hline & $2 \mathrm{~S} 1$ & ZSU234 & BRDM2 & BTR60 & BTR70 & BMP2 & D7 & ZIL131 & T62 & $\mathrm{T} 72$ & \\
\hline 2S1 & 273 & 1 & 0 & 0 & 0 & 0 & 0 & 0 & 0 & 0 & 99.64 \\
\hline ZSU234 & 0 & 274 & 0 & 0 & 0 & 0 & 0 & 0 & 0 & 0 & 100 \\
\hline BRDM2 & 0 & 0 & 272 & 1 & 1 & 0 & 0 & 0 & 0 & 0 & 99.27 \\
\hline BTR60 & 0 & 0 & 6 & 180 & 6 & 1 & 0 & 0 & 0 & 0 & 93.26 \\
\hline BTR70 & 0 & 0 & 0 & 10 & 182 & 0 & 0 & 0 & 0 & 0 & 94.79 \\
\hline BMP2 & 0 & 0 & 6 & 3 & 7 & 179 & 0 & 0 & 0 & 0 & 91.79 \\
\hline D7 & 0 & 0 & 0 & 0 & 0 & 0 & 273 & 0 & 1 & 0 & 99.64 \\
\hline ZIL131 & 0 & 0 & 0 & 0 & 0 & 0 & 0 & 274 & 0 & 0 & 100 \\
\hline T62 & 0 & 0 & 0 & 0 & 0 & 0 & 0 & 0 & 273 & 0 & 100 \\
\hline $\mathrm{T} 72$ & 0 & 0 & 0 & 0 & 0 & 0 & 0 & 0 & 0 & 274 & 100 \\
\hline Total & & & & & & & & & & & 97.84 \\
\hline
\end{tabular}


Table 3: The experimental results with the method in Literature [19]

\begin{tabular}{|c|c|c|c|c|c|c|c|c|c|c|c|}
\hline \multirow{2}{*}{ Types } & \multicolumn{2}{|c|}{ artillery } & \multicolumn{6}{|c|}{ truck } & \multicolumn{2}{|c|}{$\operatorname{tank}$} & \multirow{2}{*}{$P_{c c}(\%)$} \\
\hline & $2 \mathrm{~S} 1$ & ZSU234 & BRDM2 & BTR60 & BTR70 & BMP2 & D7 & ZIL131 & T62 & T72 & \\
\hline $2 \mathrm{~S} 1$ & 190 & 1 & 9 & 5 & 5 & 14 & 0 & 21 & 7 & 22 & 69.3 \\
\hline ZSU234 & 1 & 249 & 1 & 3 & 0 & 1 & 4 & 6 & 2 & 7 & 90.8 \\
\hline BRDM2 & 3 & 0 & 220 & 6 & 18 & 9 & 0 & 15 & 1 & 2 & 80.2 \\
\hline BTR60 & 4 & 0 & 11 & 168 & 4 & 0 & 4 & 1 & 1 & 2 & 86.1 \\
\hline BTR70 & 4 & 0 & 4 & 3 & 181 & 3 & 0 & 1 & 0 & 0 & 92.3 \\
\hline BMP2 & 4 & 4 & 9 & 2 & 9 & 157 & 0 & 6 & 0 & 4 & 80.5 \\
\hline D7 & $\mathbf{0}$ & 7 & 0 & 0 & 0 & 0 & 252 & 5 & 8 & 2 & 91.9 \\
\hline ZIL131 & 12 & 0 & 6 & 5 & 7 & 5 & 1 & 226 & 3 & 9 & 82.4 \\
\hline T62 & 7 & 2 & 1 & 5 & 0 & 2 & 4 & 7 & 242 & 3 & 88.6 \\
\hline $\mathrm{T} 72$ & 8 & 1 & 3 & 1 & 1 & 3 & 0 & 9 & 2 & 168 & 85.7 \\
\hline Total & & & & & & & & & & & 84.7 \\
\hline
\end{tabular}

Table 4: The experimental results of our method under SOC_2

\begin{tabular}{|c|c|c|c|c|c|c|c|c|c|c|c|}
\hline \multirow{2}{*}{ Types } & \multicolumn{2}{|c|}{ artillery } & \multicolumn{6}{|c|}{ truck } & \multicolumn{2}{|c|}{$\operatorname{tank}$} & \multirow{2}{*}{$P_{c c}(\%)$} \\
\hline & $2 \mathrm{~S} 1$ & ZSU234 & BRDM2 & BTR60 & BTR70 & BMP2 & D7 & ZIL131 & T62 & T72 & \\
\hline 2S1 & 274 & 0 & 0 & 0 & 0 & 0 & 0 & 0 & 0 & 0 & 100 \\
\hline ZSU234 & 0 & 274 & 0 & 0 & 0 & 0 & 0 & 0 & 0 & 0 & 100 \\
\hline BRDM2 & 0 & 0 & 269 & 0 & 0 & 5 & 0 & 0 & 0 & 0 & 98.18 \\
\hline BTR60 & 0 & 0 & 5 & 188 & 2 & 0 & 0 & 0 & 0 & 0 & 96.42 \\
\hline BTR70 & 0 & 0 & 1 & 1 & 193 & 1 & 0 & 0 & 0 & 0 & 98.47 \\
\hline BMP2 & 0 & 0 & 0 & 1 & 2 & 192 & 0 & 0 & 0 & 0 & 98.46 \\
\hline D7 & 0 & 0 & 0 & 0 & 0 & 0 & 274 & 0 & 0 & 0 & 100 \\
\hline ZIL131 & 0 & 0 & 0 & 0 & 0 & 0 & 0 & 274 & 0 & 0 & 100 \\
\hline T62 & 0 & 0 & 0 & 0 & 0 & 0 & 0 & 0 & 273 & 0 & 100 \\
\hline T72 & 0 & 0 & 0 & 0 & 0 & 0 & 0 & 0 & 0 & 274 & 100 \\
\hline Total & & & & & & & & & & & 99.15 \\
\hline
\end{tabular}

Table 5: The experimental results with the method in Literature [27]

\begin{tabular}{|c|c|c|c|c|c|c|c|c|c|c|c|}
\hline \multirow{2}{*}{ Types } & \multicolumn{2}{|c|}{ artillery } & \multicolumn{6}{|c|}{ truck } & \multicolumn{2}{|c|}{$\operatorname{tank}$} & \multirow{2}{*}{$P_{c c}(\%)$} \\
\hline & $2 \mathrm{~S} 1$ & ZSU234 & BRDM2 & BTR60 & BTR70 & BMP2 & D7 & ZIL131 & T62 & T72 & \\
\hline $2 \mathrm{~S} 1$ & 266 & 0 & 0 & 2 & 1 & 2 & 0 & 0 & 0 & 3 & 97.08 \\
\hline ZSU234 & 0 & 271 & 0 & 0 & 0 & 0 & 3 & 0 & 0 & 0 & 98.91 \\
\hline BRDM2 & 0 & 0 & 271 & 0 & 0 & 0 & 0 & 3 & 0 & 0 & 98.91 \\
\hline BTR60 & 0 & 4 & 3 & 177 & 7 & 0 & 0 & 0 & 0 & 4 & 90.77 \\
\hline BTR70 & 0 & 0 & 0 & 2 & 193 & 0 & 0 & 0 & 0 & 1 & 98.47 \\
\hline BMP2 & 1 & 0 & 0 & 4 & 5 & 547 & 0 & 0 & 0 & 30 & 93.19 \\
\hline D7 & 0 & 0 & 0 & 0 & 0 & 0 & 273 & 1 & 0 & 0 & 99.64 \\
\hline ZIL131 & 1 & 0 & 0 & 0 & 0 & 0 & 1 & 272 & 0 & 0 & 99.27 \\
\hline T62 & 1 & 0 & 0 & 0 & 0 & 0 & 2 & 0 & 268 & 2 & 98.17 \\
\hline $\mathrm{T} 72$ & 1 & 0 & 0 & 0 & 0 & 0 & 0 & 0 & 0 & 581 & 99.83 \\
\hline Total & & & & & & & & & & & 97.58 \\
\hline
\end{tabular}


Table 6: The training and testing data of EOC_1

\begin{tabular}{|c|c|c|c|c|c|c|c|}
\hline \multirow{2}{*}{ Type } & \multicolumn{3}{|c|}{ Training set } & \multicolumn{3}{|c|}{ Test set } & \multirow{2}{*}{ Image size } \\
\hline & variant & depression angle & number & variant & depression angle & number & \\
\hline 2S1 & B_01 & $17^{\circ}$ & $299 * 3000$ & B_01 & $30^{\circ}$ & 288 & $64 * 64$ \\
\hline ZSU234 & D_08 & $17^{\circ}$ & $299 * 3000$ & D_08 & $30^{\circ}$ & 288 & $64 * 64$ \\
\hline BRDM2 & E_71 & $17^{\circ}$ & $298 * 3000$ & E_71 & $30^{\circ}$ & 287 & $64 * 64$ \\
\hline T72 & \#A64 & $\begin{array}{c}17^{\circ} \\
\text { sum: } 1195 * 3000\end{array}$ & $299 * 3000$ & \#A64 & $\begin{array}{c}30^{\circ} \\
\text { sum: } 1151\end{array}$ & 288 & $64 * 64$ \\
\hline
\end{tabular}

Table 7: The experimental results of our method under EOC_1

\begin{tabular}{|c|c|c|c|c|c|}
\hline \multirow{2}{*}{ Type } & \multicolumn{2}{|c|}{ artillery } & \multirow{2}{*}{$\begin{array}{c}\text { truck } \\
\text { BRDM2 }\end{array}$} & \multirow{2}{*}{$\begin{array}{l}\operatorname{tank} \\
\mathrm{T} 72\end{array}$} & \multirow{2}{*}{$P_{c c}(\%)$} \\
\hline & $2 \mathrm{~S} 1$ & ZSU234 & & & \\
\hline $2 \mathrm{~S} 1$ & 288 & 0 & 0 & 0 & 100 \\
\hline ZSU234 & 5 & 225 & 0 & 0 & 98.26 \\
\hline BRDM2 & 0 & 0 & 287 & 0 & 100 \\
\hline T72 & 0 & 0 & 0 & 288 & 100 \\
\hline Total & & & & & 99.57 \\
\hline
\end{tabular}

Table 8: The training and testing set of EOC_2

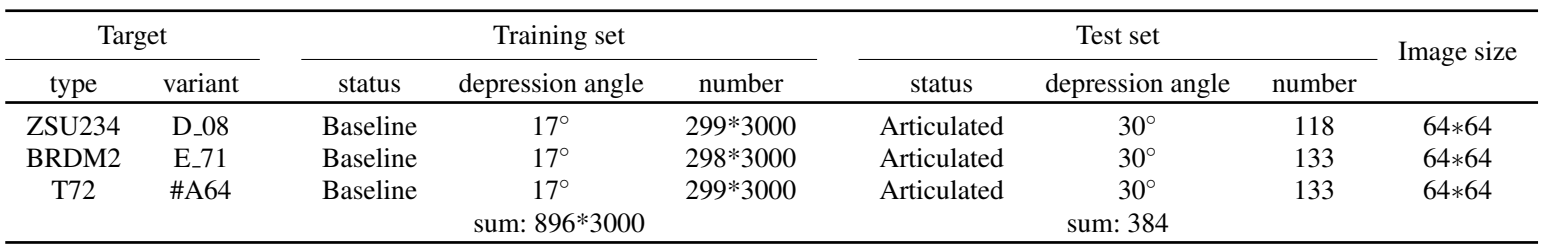

Table 9: The experimental results of our method under EOC_2

\begin{tabular}{|c|c|c|c|c|}
\hline \multirow{2}{*}{ Type } & artillery & trucks & tank & \multirow{2}{*}{$P_{c c}(\%)$} \\
\hline & ZSU234 & BRDM2 & $\mathrm{T} 72$ & \\
\hline ZSU234 & 118 & 0 & 0 & 100 \\
\hline BRDM2 & 0 & 133 & 0 & 100 \\
\hline T72 & 0 & 0 & 133 & 100 \\
\hline Total & & & & 100 \\
\hline
\end{tabular}

Table 10: The training and testing set data of EOC_3

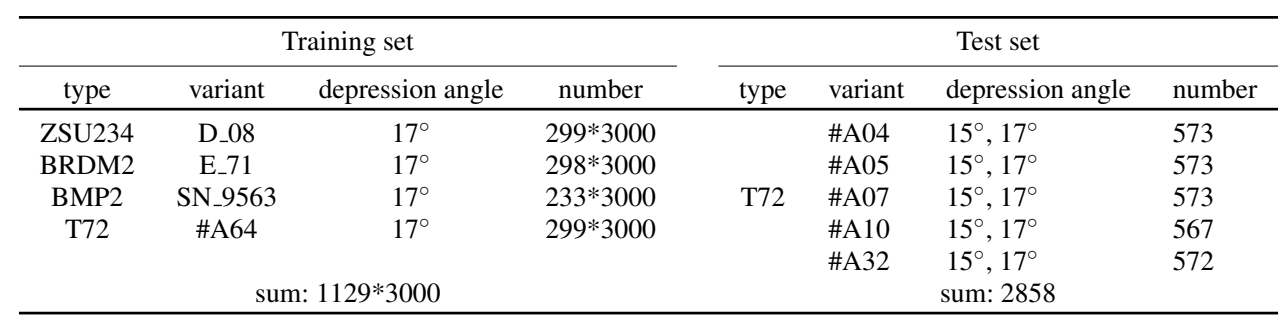


Table 11: The experimental results of our method under EOC_3

\begin{tabular}{|c|c|c|c|c|c|}
\hline \multirow{2}{*}{$\begin{array}{c}\mathrm{T} 72 \\
\text { variant }\end{array}$} & \multirow{2}{*}{$\begin{array}{l}\text { artillery } \\
\text { ZSU234 }\end{array}$} & \multicolumn{2}{|c|}{ trucks } & \multirow{2}{*}{$\begin{array}{l}\operatorname{tank} \\
\mathrm{T} 72 \\
\end{array}$} & \multirow{2}{*}{$P_{c c}(\%)$} \\
\hline & & BMP2 & BRDM2 & & \\
\hline \#A04 & 0 & 0 & 0 & 573 & 100 \\
\hline \#A05 & 0 & 0 & 0 & 573 & 100 \\
\hline \#A07 & 0 & 0 & 0 & 573 & 100 \\
\hline \#A10 & 0 & 0 & 0 & 567 & 100 \\
\hline \#A32 & 0 & 0 & 0 & 572 & 100 \\
\hline Total & & & & & 100 \\
\hline
\end{tabular}

Table 12: Training set and test set parameter configuration (EOC_4) for the experiment on the classification of mixed depression angle of variant of multi models and multi targets

\begin{tabular}{|c|c|c|c|c|c|c|c|}
\hline \multicolumn{4}{|c|}{ Training set } & \multicolumn{4}{|c|}{ Test set } \\
\hline type & variant & depression angle & number & type & variant & depression angle & number \\
\hline ZSU234 & D_08 & $17^{\circ}$ & $299 * 3000$ & \multirow{2}{*}{ BMP2 } & SN_9566 & $15^{\circ}, 17^{\circ}$ & 428 \\
\hline BRDM2 & E_71 & $17^{\circ}$ & $298 * 3000$ & & C_21 & $15^{\circ}, 17^{\circ}$ & 429 \\
\hline BMP2 & SN_9563 & $17^{\circ}$ & $233 * 3000$ & \multirow{7}{*}{ T72 } & \#A04 & $15^{\circ}, 17^{\circ}$ & 573 \\
\hline $\mathrm{T} 72$ & \#A64 & $17^{\circ}$ & $299 * 3000$ & & \#A05 & $15^{\circ}, 17^{\circ}$ & 573 \\
\hline & & & & & \#A07 & $15^{\circ}, 17^{\circ}$ & 573 \\
\hline & & & & & \#A10 & $15^{\circ}, 17^{\circ}$ & 567 \\
\hline & & & & & \#A32 & $15^{\circ}, 17^{\circ}$ & 572 \\
\hline & & & & & \#A62 & $15^{\circ}, 17^{\circ}$ & 573 \\
\hline & & & & & \#A63 & $15^{\circ}, 17^{\circ}$ & 573 \\
\hline \multicolumn{4}{|c|}{ sum: $1129 * 3000$} & \multicolumn{4}{|c|}{ sum: 4861} \\
\hline
\end{tabular}

Table 13: The experimental results of our method under EOC_4

\begin{tabular}{|c|c|c|c|c|c|c|}
\hline \multicolumn{2}{|c|}{ Type } & \multirow{2}{*}{$\begin{array}{c}\text { artillery } \\
\text { ZSU234 }\end{array}$} & \multicolumn{2}{|c|}{ trucks } & \multirow{2}{*}{$\begin{array}{c}\text { tanks } \\
\text { T72 }\end{array}$} & \multirow{2}{*}{$P_{c c}(\%)$} \\
\hline top & variant & & BMP2 & BRDM2 & & \\
\hline \multirow[t]{2}{*}{ BMP2 } & SN_9566 & 0 & 423 & 4 & 0 & 99.06 \\
\hline & C_21 & 0 & 426 & 3 & 0 & 99.3 \\
\hline \multirow[t]{7}{*}{ T72 } & \#A04 & 0 & 0 & 0 & 573 & 100 \\
\hline & \#A05 & 0 & 0 & 0 & 573 & 100 \\
\hline & \#A07 & 0 & 0 & 0 & 573 & 100 \\
\hline & \#A10 & 0 & 0 & 0 & 567 & 100 \\
\hline & \#A32 & 0 & 0 & 0 & 572 & 100 \\
\hline & \#A62 & 0 & 0 & 0 & 573 & 100 \\
\hline & \#A63 & 0 & 0 & 0 & 573 & 100 \\
\hline Total & & & & & & 99.82 \\
\hline
\end{tabular}

Table 14: Comparison sheet of classification accuracy between Our method and other algorithms

\begin{tabular}{ccc}
\hline Model & SOC(\%) & EOC_1(\%) \\
\hline EMACH [32] & 88 & 77 \\
IGT [33] & 95 & 85 \\
MSRC [34] & 93.6 & 98.4 \\
MSS [35] & 96.6 & 98.2 \\
M-PMC [36] & 98.8 & 98.2 \\
Our method & $\mathbf{9 9 . 0 4}$ & $\mathbf{9 9 . 5 7}$ \\
\hline
\end{tabular}


Table 15: "CNN+Classifier" model parameter configuration

\begin{tabular}{|c|c|c|c|}
\hline Softmax & RandomForest & AdaBoost & Bagging \\
\hline \multicolumn{4}{|c|}{ Conv.10@11 } \\
\hline \multicolumn{3}{|c|}{ Conv.120@22 } \\
\hline \multicolumn{3}{|c|}{ Conv.120@ 55 + Max-pooling@ @22 } \\
\hline \multicolumn{3}{|c|}{ Conv.18@99 + Max-pooling@77 } \\
\hline A & B & C & D \\
\hline Convolutional neural network configuration \\
\hline
\end{tabular}

Table 16: Comparison of experimental results of "CNN+Classifier" models in Table 15 under SOC and EOC

\begin{tabular}{cccccc}
\hline Model & SOC_2(\%) & EOC_1 $(\%)$ & EOC_2(\%) & EOC_3(\%) & EOC_4(\%) \\
\hline A & 98.58 & 96.55 & 99.05 & 99.13 & 98.98 \\
B & 98.74 & 95.36 & 99.11 & 99.04 & 98.25 \\
C & 98.13 & 94.32 & 98.24 & 98.14 & 97.33 \\
D & 98.29 & 95.67 & 98.04 & 97.04 & 98.08 \\
Our method & $\mathbf{9 9 . 1 5}$ & $\mathbf{9 9 . 5 7}$ & $\mathbf{1 0 0}$ & $\mathbf{1 0 0}$ & $\mathbf{9 9 . 8 2}$ \\
\hline
\end{tabular}


Figures

List of Figures

1 The proposed DCNN+SVM model . . . . . . . . . . . . . . . . . . . . 15

2 Various variants included in the MSTAR database . . . . . . . . . . . . . . . . 16

3 SAR image samples and corresponding optical images of ten types of targets in the MSTAR

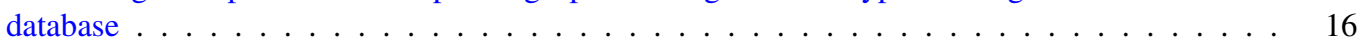

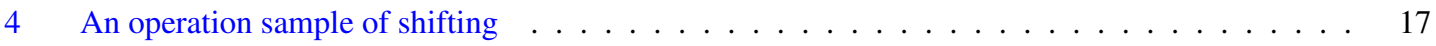

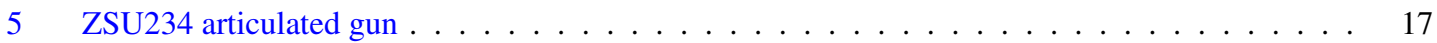

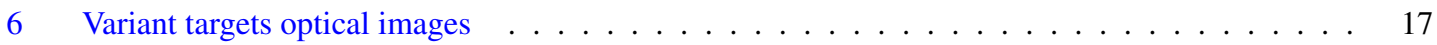

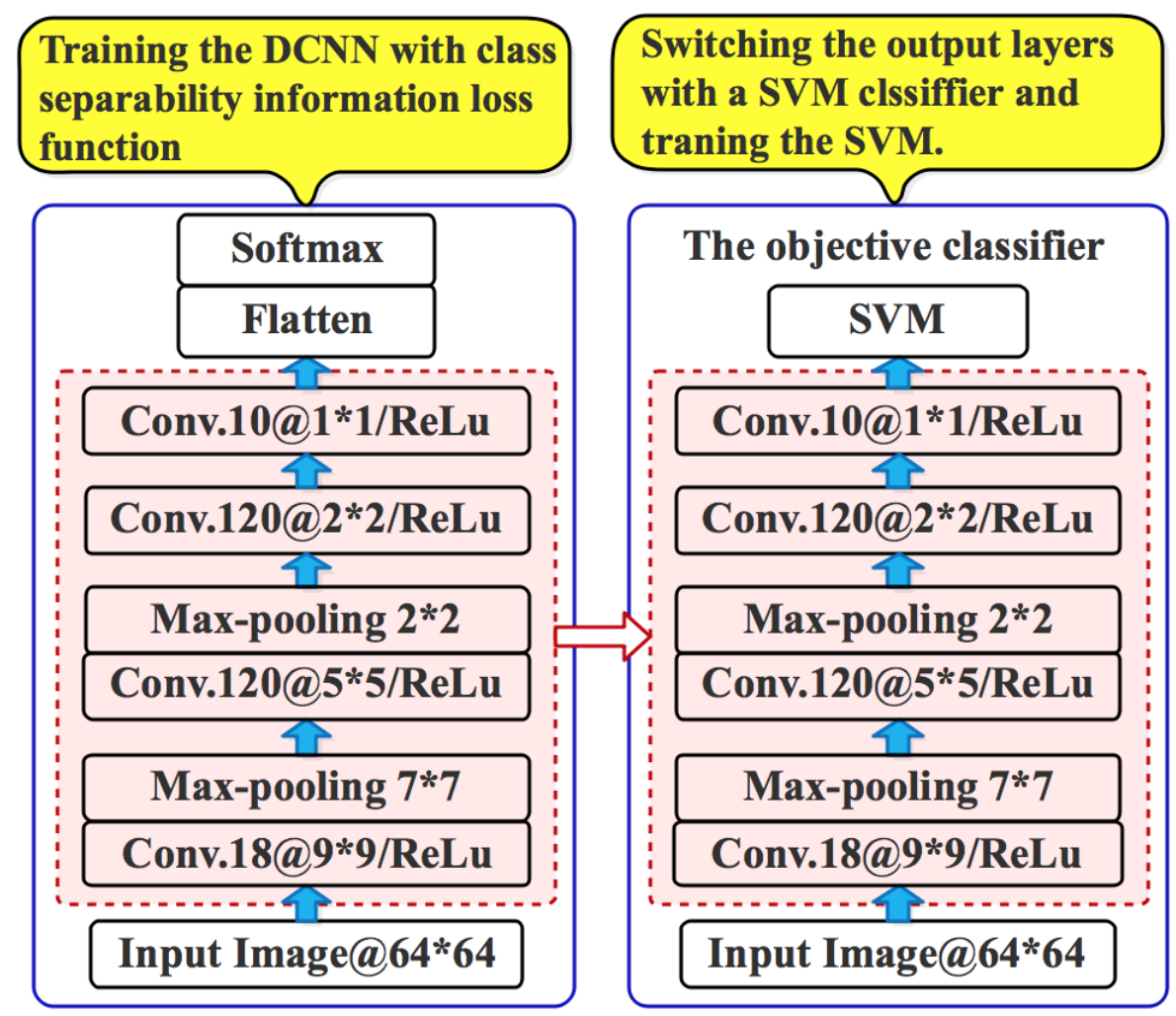

Fig. 1: The proposed DCNN+SVM model 


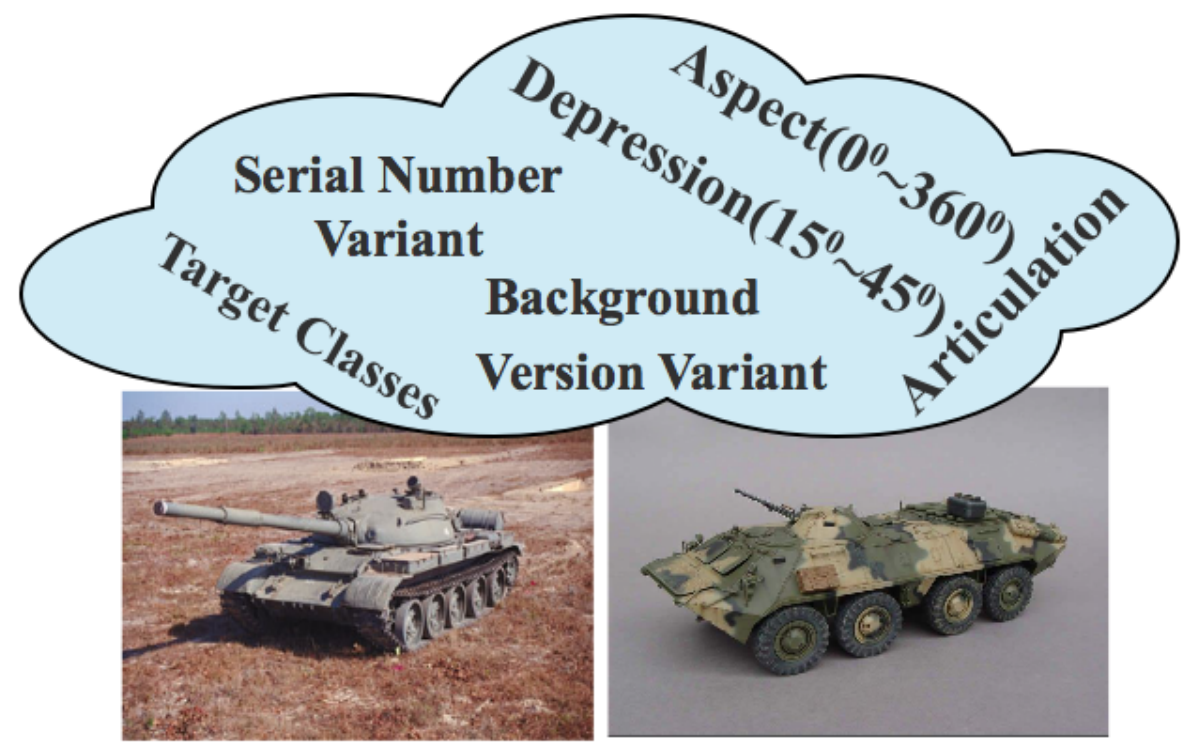

Fig. 2: Various variants included in the MSTAR database

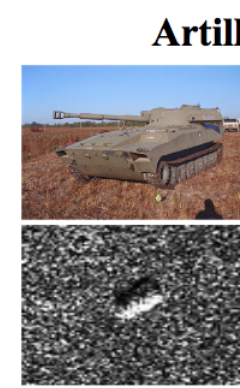

(0)2S1
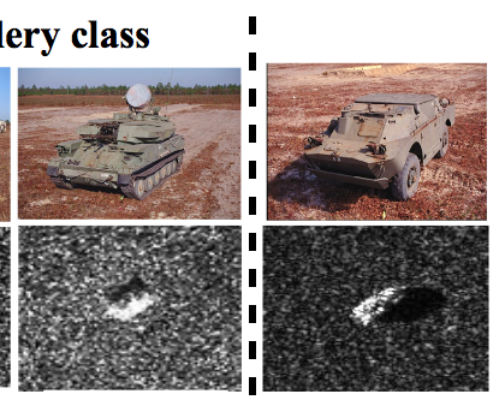

Truck class

(1)ZSU234 , (2)BRDM-2

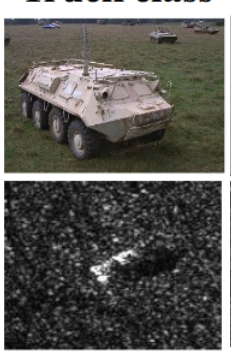

(3)BTR60

Tank class

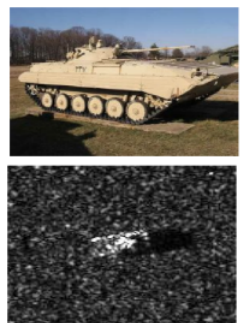
Truck class
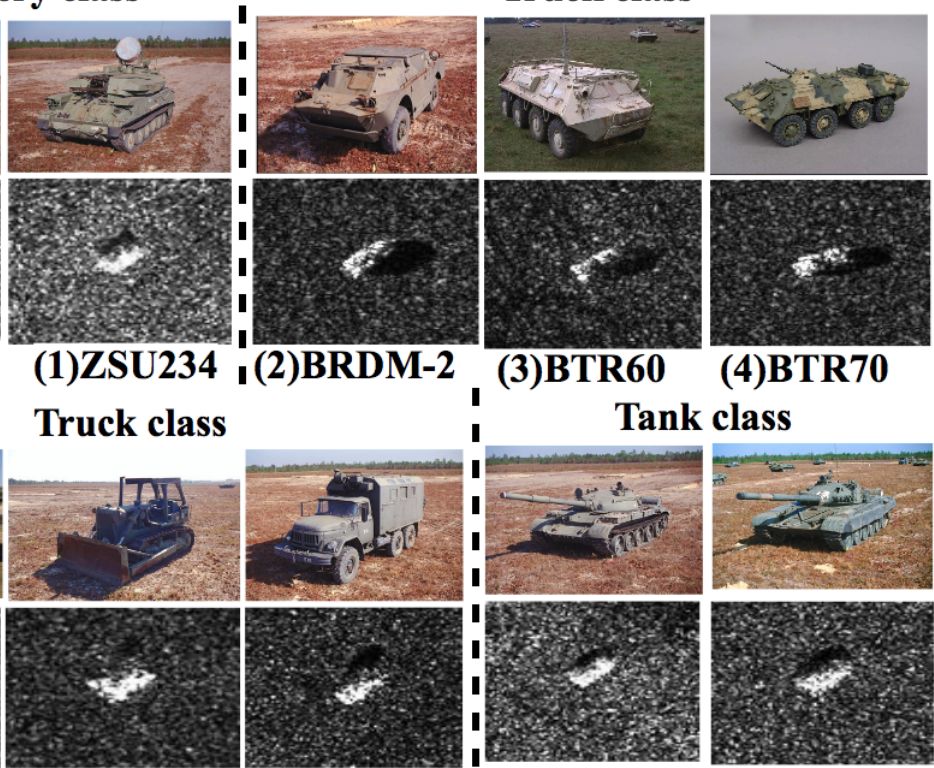

(5)BMP2

(6)D7

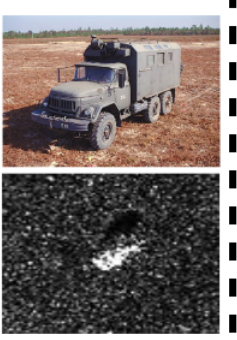

(7)ZIL131

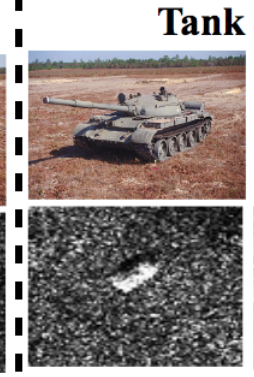

(8)T62

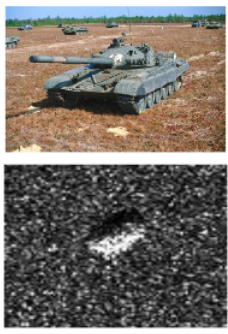

(9)T72

Fig. 3: SAR image samples and corresponding optical images of ten types of targets in the MSTAR database 


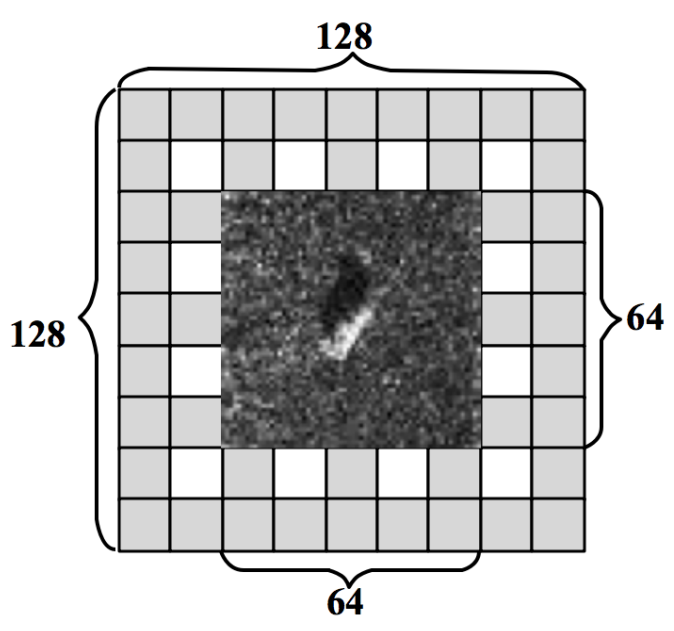

(a) Computing target translation

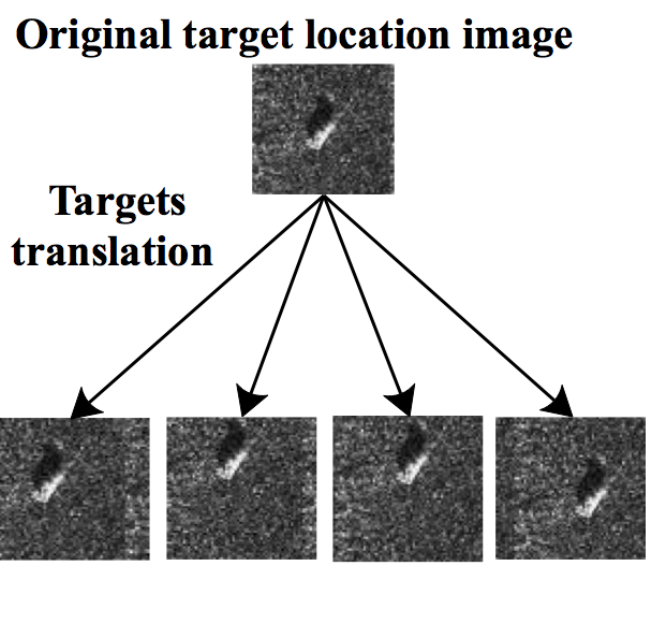

(b) Result of target translation

Fig. 4: An operation sample of shifting

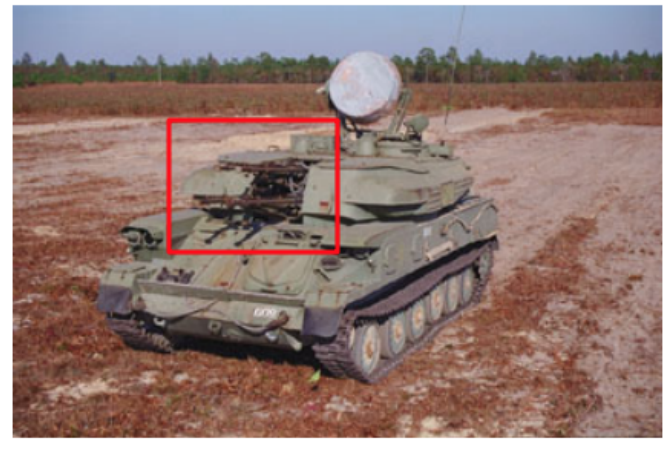

(a) Turret straight

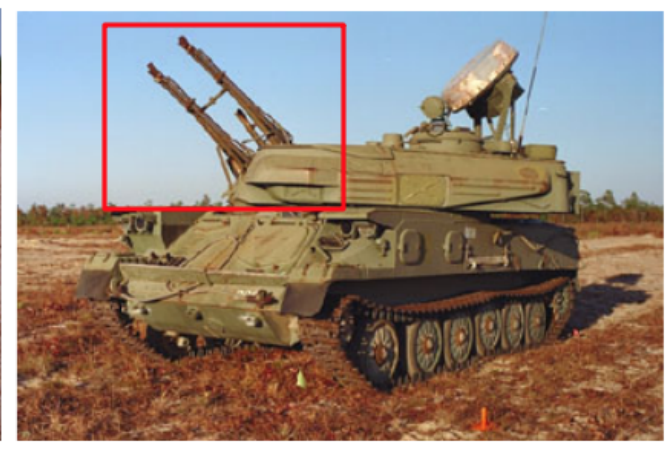

(b) Turret articulated

Fig. 5: ZSU234 articulated gun

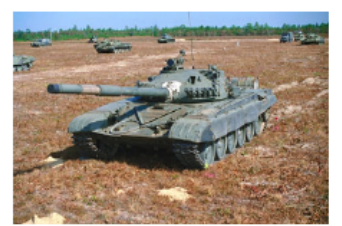

T72\#04

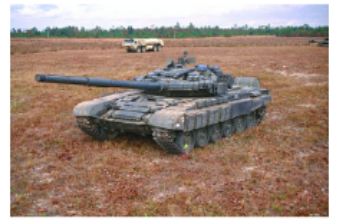

T72\#32

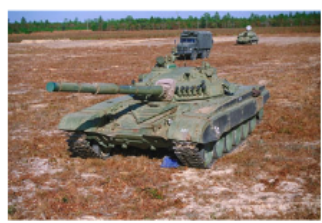

T72\#05

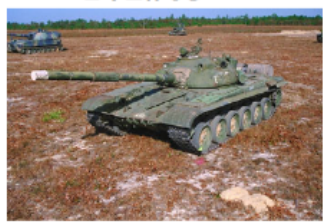

T72\#62

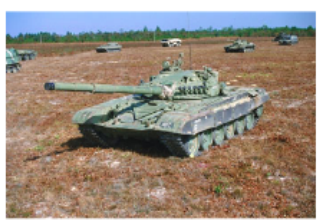

T72\#07

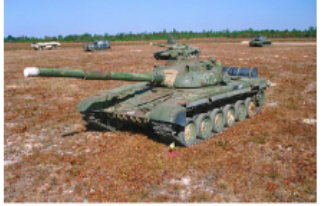

T72\#63

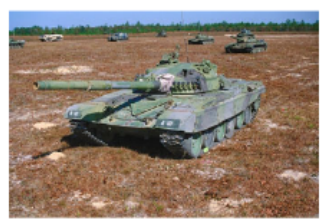

T72\#10

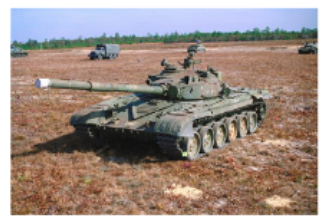

T72\#64

Fig. 6: Variant targets optical images 